\title{
Low-level laser therapy and exercise for patients with shoulder disorders in physiotherapy practice (a systematic review protocol)
}

\author{
Adedapo W Awotidebe ${ }^{1 *}$, Gakeemah Inglis-Jassiem² and Taryn Young ${ }^{3}$
}

\begin{abstract}
Background: Low-level laser therapy is one of the adjunct treatments of choice with exercise therapy for shoulder rehabilitation in physiotherapy clinical practices. Although previous reviews have found little use of low-level laser therapy, there are recent trials whose findings are yet to be systematically reviewed.

Methods: We plan to do a systematic review to assess the effects of low-level laser therapy with exercise and exercise alone in participants who are 18 years and above, with a clinical or radiological diagnosis of various shoulder pathologies. We will search CENTRAL, MEDLINE, CINAHL, PEDro, Science Direct, Scopus and Physiotherapy Choices regardless of publication status. We will hand search for subject-specific journals (PhotoMedicine and Laser Surgery, Lasers in Surgery and Medicine and Journals of Lasers in Medical Science) and conference proceedings of World Association for Laser Therapy. Two review authors will independently screen, select studies, extract data and assess the risk of bias based on a priori criteria. Disagreements between review authors will be resolved either through discussion or consultation with a third review author. If there are at least two clinically homogeneous studies, we will perform meta-analysis.
\end{abstract}

Discussion: The findings will shed more light on the benefit of low-level laser therapy as an adjunct treatment to exercise in the management of shoulder disorders. The findings may also inform decision makers in the review and development of guidelines for shoulder rehabilitation in physiotherapy practices.

Systematic review registration: PROSPERO CRD42014013691

Keywords: Low-level laser therapy, Exercise, Physiotherapy, Rehabilitation, Shoulder disorders, Systematic review

\section{Background}

Various shoulder disorders such as sub-acromial impingement, rotator-cuff tear and frozen shoulder are important complaints of shoulder pain and disability and commonest referrals to physiotherapy clinics [1]. Shoulder complaints presenting as painful shoulder, muscular weakness, diminished range of motion and restricted daily activities are reasons for outpatient visits in physiotherapy clinics [1-5]. Physiotherapy is considered the first line of conservative management and inevitably an adjunct treatment for post-surgical interventions [6]. Of

\footnotetext{
* Correspondence: awotidebe.adedapo@gmail.com

'Division of Community Health, Department of Interdisciplinary Health,

Faculty of Medicine and Health Sciences, Stellenbosch University, Francie van Zijl Rylaan Drive, Tygerberg Campus, PO Box 19063, Cape Town 7505, South Africa

Full list of author information is available at the end of the article
}

all the shoulder complaints treated by the general practitioners (GP) in primary care, between $10 \%$ and $30 \%$ are referred for physical therapy $[1,7]$.

In a routine physiotherapy clinic, management of various musculoskeletal shoulder disorders is multifaceted and treatment includes a broad spectrum of physiotherapeutic options such as progressive strengthening exercises, strapping, electrotherapy, low-grade joint manipulation/ mobilisation therapy, acupuncture, advice and education $[7,8]$. However, compared to other treatment options, exercise therapy which includes joint and soft-tissue mobilisation, stretching/flexibility, range of motion and gradual strengthening exercises is the mainstay of rehabilitation protocol for shoulder pain [8,9]. In addition to exercise therapy, the use of low-level laser therapy (LLLT) is also evident among physiotherapists in a wide range of 
musculoskeletal injuries [10-12]. The application of LLLT is non-thermal and is considered safe; however, its use is contraindicated in part or entirely in patients with malignant tumours, epilepsy, light hypersensitivity, applications over thyroid gland, thrombotic deep vein thrombotic area and treatment around developing foetus in pregnant women [12,13]. Similarly, it is contraindicated in patients using steroids because it hinders the effect of laser treatment [14].

The LLLT, also known as phototherapy, is a noninvasive application of non-thermal and low-powered laser light of single wavelength of classes IIIa and IIIb [15]. The protocol for laser treatment in musculoskeletal disorders is associated with better performance in wavelengths of between 632 and 1,064 $\mathrm{nm}$ and laser power output of less than $5 \mathrm{~mW}$ (class IIIa) or less than $500 \mathrm{~mW}$ (class IIIb) [12]. The LLLT is typically administered in a routine therapeutic treatment as either stationary in skin contact or stationary with distance from skin over a maximum of ten trigger or painful points for $90 \mathrm{~s}$ at $2,000 \mathrm{~Hz}$ [16], $150 \mathrm{~s}$ at 3,500 $\mathrm{Hz}$ [17], or $5 \mathrm{~min}$ at $1,000 \mathrm{~Hz}[18]$ per painful point.

Mechanism of action and clinical effect of low-level laser therapy was described by Moshkovska and Mayberry [13] and illustrated in Table 1. All forms of LLLT are used on the site of pathology (for example tendon, joint capsule, cartilage, muscle) or nerve supplying area on acupuncture or trigger points. There was evidence that LLLT in vitro studies facilitated the stimulation of fibroblasts and collagen synthesis in connective tissue repair $[19,20]$. Evidence exists that LLLT, in addition to connective tissue repair, reduces inflammation and modulates pain [10] through reduction of prostaglandin $\mathrm{E}_{2}\left(\mathrm{PGE}_{2}\right)$ receptor concentration and inhibition of cyclooxygenase-2 (COX-2) $[21,22]$. Findings from animal study showed that low-power laser radiation $(780 \mathrm{~nm})$ on injured rat sciatic nerve accelerates peripheral nerve regeneration by enhancing axonal growth [23].
In recent years, low-level laser therapy is increasingly being combined with exercise than exercise alone for management of pain and functions in various rehabilitation of musculoskeletal injuries [24-26]. In spite of the drive for evidence-based practice in physiotherapy, recommending for widespread use of LLLT, especially in shoulder rehabilitation, is ambivalent because there was insufficient evidence to determine that LLLT contributes to pain relief and increased function [27]. Subsequently, findings from a number of trials on the effectiveness of LLLT on shoulder disorders are not clear-cut. Both positive results [28,29] and no-effect results [30] exist for studies targeting the benefits of LLLT. Although the physiotherapy management of various shoulder complaints is multifaceted, exercise therapy remains the mainstay of treatment $[8,31]$. For ethical reasons, in studies that evaluated the effect of LLLT, patients also received exercise or other physiotherapeutic treatments in conjunction with laser treatment $[16,18,32-34]$. It is therefore unclear what specific contribution the LLLT had on shoulder pain, range of motion and physical function. With this perspective, meta-analysis of low-level laser therapy in shoulder disorders over placebo-only treatment would have been appropriate save for the ethical considerations. However, to meet the growing demand of health practitioners and decision makers on evidence regarding LLLT, Green and colleagues reported that evidence is very low on the effectiveness of LLLT on shoulder disorders [27]. The four primary studies reviewed by Green et al. [27], England et al. [28], Saunders et al. [29], Vecchio et al. [30], and Tavena [35] were fraught with methodological limitations. Since, additional studies have examined the effectiveness of LLLT on shoulder disorders [16-18,33,36-38]. Recently, one review study [39] examined the effect of LLLT combined with an exercise programme in adults with shoulder pain. The authors did literature appraisal of four of the above studies $[16,32,33,37]$. This study had several limitations: there was no information on the assessment of risk of bias

Table 1 Mechanism of action of low-level laser therapy [13]

\begin{tabular}{lll}
\hline Types of effect & Mechanisms & Outcomes \\
\hline Anti-inflammatory effect & Activation of microcirculation & Elimination of oedema \\
& Prostaglandin level changes & \\
& Equalisation of osmotic pressure & Reduction of lipid peroxidation \\
Stabilisation of lipid peroxidation & Reactivation of superoxide dysmutase and catalase & Increase pain threshold \\
Analgesic & Activation of neuron metabolism and endorphin level growth & Defective epithelisation \\
Reparation of wound stimulation & ATP accumulation & Protein and collagen synthesis \\
& Activation of cellular metabolism & Capillary formation \\
Immune response stimulation & Increase proliferation of fibroblasts and other cells & \\
Reflexogenic effect & Increase proliferation of immune modifying cells & Stimulation of physiological functions \\
\hline
\end{tabular}


of the included studies, and this is likely to affect the validity of findings. Moreover, the authors reported a qualitative summary of individual studies to draw conclusions; a meta-analysis of these studies would have provided a more precise estimate of the effects of LLLT on shoulder pain and function. In addition, undertaking subgroup and sensitivity analyses could have impact on the conclusions. It is therefore important and inevitable to conduct systematic reviews and meta-analysis of all these trials.

\section{Objectives}

The aim of this systematic review is to assess the effects of low-level laser therapy with exercise compared to exercise alone in the treatment of shoulder disorders.

The secondary objective is to assess the safety of lowlevel laser therapy in the treatment of shoulder disorders.

\section{Methods/Design}

\section{Criteria for considering studies for this review} Types of studies

The review will consider randomised controlled trials (RCTs).

\section{Types of participants}

The review will consider participants who are 18 years and above, with a clinical or radiological diagnosis of various shoulder pathologies by the referring orthopaedic surgeons and physiotherapist and receiving treatments in both in- and outpatient physiotherapy clinics.

\section{Types of interventions}

The intervention group should have received exercise therapy and either class IIIa (laser power output of less than $5 \mathrm{~mW}$ ) or IIIb (laser power output of less than $500 \mathrm{~mW}$ ) LLLT at the site of pathology within the required range of laser treatment protocol for musculoskeletal injuries. The control group should have received exercise therapy and placebo LLLT, and trials that do not have control groups but used one shoulder as treatment and opposite shoulder as a control will be excluded to avoid co-intervention of effect. Participants in the eligible studies would have received LLLT as part of treatment for included shoulder pathologies and information on duration, intensity, dosage, frequency, treatment time and accumulated energy delivered from all sessions must be reported.

\section{Types of outcome measures}

The primary outcomes will be pain, disability and range of motion related to shoulder injury.

- Pain measured by visual analogue scale (VAS) and other categorical rating scales (the higher the rating, the higher the pain).
- Disability: measures will include the different validated instruments of disability (Shoulder Pain and Disability Index, pain-free muscle strength, 6-minute maximal walking distance, Western Ontario and McMasters Universities Osteoarthritis Index, Roland Morris Disability Index, Oswestry Pain and Disability Index). The mean (standard deviation) disability scores will be used to pool weighted mean differences (if one disability scale is used in all the included studies). Where studies have different scales to measure disability, standardised mean differences (SMDs) will be used.

- Joint range of motion (passive and active).

The secondary outcome measures will include:

- Adverse effect measured by the number of patients experiencing untoward reactions following LLLT treatment.

- Other pain outcomes (for example pain measured at rest, pain on palpation and during physical activities).

- Quality of life measured by Short Form-36 (SF-36).

\section{Search methods for identification of studies Databases}

To identify eligible RCTs for this review, an exhaustive search of the following subject-specific databases will be searched:

- Cochrane Central Register of Controlled Trials (CENTRAL)

- MEDLINE (via EBSCOhost) (1996 to date)

- CINAHL (via EBSCOhost) (1996 to date)

- PEDro (Physiotherapy Evidence Database) (1997 to date)

- Science Direct

- Scopus

- Physiotherapy Choices

There was no known information on when LLLT was introduced as a treatment of choice in physiotherapy practice. However, we commenced the search from 1996 because all the included studies in a previous and related systematic review [27] were published on or before 1995. The search strategy for MEDLINE is detailed in Additional file 1 and will be adapted for other databases.

\section{Conference proceedings}

We will search conference proceedings of World Association for Laser Therapy to identify other relevant studies for inclusion in this review. We will also send emails to all the invited speakers, oral and poster speakers in the last World Association for Laser Therapy 2012 Conference 
requesting for information on additional unpublished trials relevant to this review.

\section{Searching other resources}

Hand searching of subject-specific journals: PhotoMedicine and Laser Surgery (2007-2011), Lasers in Surgery and Medicine (1996 to 2014) and Journals of Lasers in Medical Science (2010 to present) will be checked manually to identify any other studies. Lastly, we will manually screen a reference list of included studies.

\section{Data collection and analysis Selection of studies}

Two review authors (AD and GIJ) will independently screen and select titles and abstracts of relevant trials. Full texts of the articles will be obtained and assessed for all potential eligible studies. These will be independently assessed to identify studies to be included in this review based on a priori inclusion criteria. We will contact the authors of primary studies if further information is needed or some data are missing. We will resolve disagreements on what studies to include in the review through discussion, and should we fail to agree, a third review author (TY) will be contacted to make the final decision on disputed studies.

\section{Data extraction and management}

The two review authors (AD and GIJ) who will select studies will independently extract the following information from each included trial:

- Characteristics of the studies including source of funding, country, setting, publication date, citation and contact details.

- Participant characteristics including age, sex, socio-demographics, diagnosis criteria, study population.

- Characteristics of LLLT in both intervention and control group, wavelength, class of laser, application procedure, power density, energy dose delivered, treatment time, number of sessions, frequency, average output of laser reported, spot size on the skin, power density and accumulated energy delivered for all sessions.

- Outcome measures for dichotomous parameters, the number of events will be extracted and means and standard deviations for continuous outcomes for estimating the effect of treatment.

Primary authors of the included studies will be contacted if further information is needed and disagreements between review authors will be resolved either through discussion or consultation with a third review author (TY). Data will be captured in the table of included studies.

\section{Assessment of risk of bias in included studies}

We will assess the risk of bias in included studies independently against the following methodological domains as recommended by the Cochrane Collaboration [40]:

- Sequence generation

- Allocation sequence concealment

- Blinding of patients, health personnel and outcome assessors

- Incomplete outcome data

- Selective outcome reporting

- Other biases

Each of these domains will be judged as: 'low risk of bias,' 'high risk of bias', or 'uncertain'. We will resolve any disagreement by discussion or arbitration through a third review author.

\section{Measurement of treatment effect}

For continuous data (for example visual analogue pain rating scale, shoulder disability assessed by validated instruments, shoulder range of motion, and Health-related quality of life), we will calculate mean differences (MD) with corresponding 95\% confidence intervals. However, we will use an SMD if different instruments were used to measure the same outcome or construct. Where possible, if shoulder pain were assessed using a binary pain rating scale (that is, pain vs. no pain), and adverse events (number of the events in the treatment group vs. control group), we will calculate risk ratios (RR) with corresponding 95\% confidence intervals.

\section{Unit of analysis}

The unit of analysis will be the patient.

\section{Dealing with missing data}

We will send an email to the respective trial author (s) to obtain the missing data. We will neither make any assumption nor will we perform imputations.

\section{Assessment of heterogeneity}

We will assess trials for clinical heterogeneity based on patient characteristics, intervention characteristics, control group and outcome measures. For heterogeneity, visual inspection of the forest plot, Chi square test and $\mathrm{I}^{2}$ statistic will be performed. Both $\mathrm{Chi}^{2}$ test and $\mathrm{I}^{2}$ statistic will be interpreted based on the guidelines recommended by the Cochrane Handbook for Systematic Review of Interventions [41]. The following values are interpreted as thus: 
For $\mathrm{Chi}^{2}$ :

- $p<0.05$ (strong likelihood of statistical heterogeneity)

- $p 0.05<0.1$ (likely to be heterogeneous)

- $p>0.1$ (not likely to be heterogeneous)

For $\mathrm{I}^{2}$ statistic:

- $0 \%-40 \%$ (might not be important)

- 30\%-60\% (moderately heterogeneous)

- $50 \%-90 \%$ (substantial heterogeneity)

- $75 \%-100 \%$ (considerable heterogeneity)

\section{Assessment of reporting bias}

We will assess the reporting bias using funnel plots if we have at least ten studies from included studies that are available for meta-analysis. Results of tests of visual inspection of the funnel plots will be interpreted on the basis of other sources of asymmetry (that is heterogeneity).

\section{Assessment of the quality of a body of evidence}

We will use GRADE approach [42] to evaluate the quality of evidence for individual outcome reported in this review against the following factors that may either increase or decrease the quality of a body of evidence:

- Limitations in the design and implementation

- Indirectness of evidence

- Unexplained heterogeneity or inconsistency of results

- Imprecision of results

- High probability of publication bias

- Presence of dose-response

Each individual outcome will be judged 'High,' Moderate', 'Low' and 'Very low' levels of quality of a body evidence.

\section{Data synthesis}

The diagnostics of shoulder problem is often problematic and clinical heterogeneity can be expected because of variations in a population's demographic characteristics and applications of interventions between studies (for example content, frequency, intensity). Therefore, we would perform meta-analysis using random effects model for estimates of intervention effects.

\section{Assessment of safety of low-level laser therapy}

We will describe the report of adverse effects or complications arising from the use of LLLT in included studies.

\section{Subgroup analysis and investigation of heterogeneity}

If there are substantial studies available for meta-analysis, we will perform subgroup analyses using RevMan to compare effect estimates to investigate whether LLLT works differently in subset of participants (young participants vs. old participants: 18-35 years vs. $>35$ years), duration of intervention (short term: $<3$ months vs. intermediate: 3-6 months vs. long term: $>6$ months) and subsets of diagnosis (for example types of shoulder disorder).

\section{Sensitivity analysis}

We will a perform sensitivity analysis to examine how methodological criteria (allocation concealment and blinding) affect the overall treatment effect. Specifically, we will exclude studies that report high/unclear risk of bias and see how it affects the overall treatment effect.

\section{Discussion}

To the best of the authors' knowledge, there are no published systematic reviews or meta-analysis that has specifically compared the effectiveness of low-laser therapy with and without exercise of the following trials on shoulder disorders (for example, an update from included studies from 1996 onwards). Although the previous systematic review found low evidence for the benefit of low-laser therapy in shoulder rehabilitation [27], we hope the review of recent trials will shed more light on the benefit of low-laser therapy as an adjunct treatment to exercise in the management of shoulder disorders. The findings may also inform decision makers in the review and development of guidelines for shoulder rehabilitation in physiotherapy practices.

\section{Additional file}

Additional file 1: MEDLINE search strategy using PubMed.

\section{Abbreviations}

LLLT: low-level laser therapy; MD: mean difference; OR: odds ratios; $\mathrm{RCT}$ : randomised controlled trial; RR: risk ratios; SMD: standardised mean difference; SPADI: shoulder pain and disability index; VAS: visual analogue scale.

\section{Competing interests}

The authors declare that they have no competing interests.

\section{Authors' contributions}

AD conceived of the study, contributed to the design of the protocol and drafted the manuscript. GIJ involved in the design of the protocol and helped to critically review the manuscript for its intellectual content. TY participated in the design of the protocol and critically reviewed the manuscript for its intellectual content. All authors (AD, GIJ, TY) read and approved the final manuscript.

\section{Acknowledgements}

The authors would like to thank Ms. Anel Schoonees from Centre for Evidence-based Health Care, Stellenbosch University, South Africa, for her assistance in developing and verifying the search strings.

\section{Author details}

${ }^{1}$ Division of Community Health, Department of Interdisciplinary Health, Faculty of Medicine and Health Sciences, Stellenbosch University, Francie van 
Zijl Rylaan Drive, Tygerberg Campus, PO Box 19063, Cape Town 7505, South Africa. ${ }^{2}$ Division of Physiotherapy, Department of Interdisciplinary Health Sciences, Faculty of Medicine and Health Sciences, Stellenbosch University, Francie van Zijl Rylaan Drive, Tygerberg Campus, PO Box 19063, Cape Town 7505, South Africa. ${ }^{3}$ Centre for Evidence-based Health Care, Faculty of Medicine and Health Sciences, Stellenbosch University, Francie van Zijl Rylaan Drive, Tygerberg Campus, PO Box 19063, Cape Town 7505, South Africa.

Received: 9 December 2014 Accepted: 17 April 2015

Published online: 30 April 2015

\section{References}

1. Kooijman M, Swinkels I, van Dijk C, Bakker D, Veenhof C. Patients with shoulder syndromes in general and physiotherapy practice: an observational study. BMC. 2013;14:128.

2. Cha J, Kim J, Hong J, Choi Y, Kim M, Cho J, et al. A 12-week rehabilitation program improves body composition, pain sensation, and internal/external torques of baseball pitchers with shoulder impingement syndrome. J Exerc Rehabil. 2014;10:35-44.

3. Karel YH, Scholten-Peeters WG, Graaf MT, Duijn E, Ottenheijm RP, van den Borne MP, et al. Current management and prognostic factors in physiotherapy practice for patients with shoulder pain: design of a prospective cohort study. BMC Musculoskelet Disord. 2013;14:62.

4. Buchbinder R, Youd JM, Green S, Stein A, Forbes A, Harris A, et al. Efficacy and cost-effectiveness of physiotherapy following glenohumeral joint distension for adhesive capsulitis: a randomised trial. Arthritis Rheum. 2007;57:1027-37

5. Ainsworth R. Physiotherapy rehabilitation in patients with massive, irreparable rotator cuff tears. Musculoskeletal Care. 2006;4:140-51.

6. Kromer TO, Tautenhahn UG, de Bie RA, Staal JB, Bastiaenen CG. Effects of physiotherapy in patients with shoulder impingement syndrome: a systematic review of the literature. J Rehabil Med. 2009;41(11):870-80.

7. Linsell L, Dawson J, Zondervan K, Rose P, Randall T, Fitpatrick R, et al. Prevalence and incidence of adults consulting for shoulder conditions in UK primary care: patterns of diagnosis and referral. Rheumatology. 2006;45:215-21.

8. Desmeules F, Cote $\mathrm{CH}$, Fremont P. Therapeutic exercise and orthopaedic manual therapy for impingement syndrome: a systematic review. Clin J Sports Med. 2003;13:176-82.

9. Ainsworth R, Lewis J. Exercise therapy for the conservative management of full thickness tears of the rotator cuff: a systematic review. Br J Sport Med. 2007;41:200-10.

10. Bjordal JM, Lopes-Martins RA, Iversen W. A randomised, controlled trial of low level laser therapy for activated Achilles tendinitis with micro-dialysis measurement of peritendinous prostaglandin E2 concentrations. Br J Sport Med. 2006;40:76-80

11. Enwemeka CS, Parker JC, Dowdy DS, Harkness EE, Sanford LE, Woodruff LD The efficacy of low-power lasers in tissue repair and pain control: a meta-analysis study. Photomed Laser Surg. 2004;22:323-9.

12. Baxter GD, Bell AJ, Allen JM, Ravey J. Low-level laser therapy: current clinical practice in Northern Ireland. Physiotherapy. 1991;77(3):171-8.

13. Moshkovska T, Mayberry J. It is time to test low level laser therapy in Great Britain. Postgrad Med J. 2005;81:436-41.

14. Lopes-Martins RA, Albertini R, Lopes-Martins PS, De Carvalho FA, Neto HC, Iversen $\mathrm{W}$, et al. Steroid receptor antagonist miferpristone inhibits the anti-inflammatory effects of photoradiation. Photomed Laser Surg. 2006;24:197-201.

15. World Association of Laser Therapy. Consensus agreement on the design and conduct of clinical studies with low-level laser therapy and light therapy for musculoskeletal pain and disorders. PhotoMed Laser Surg. 2006;24(6):761-2.

16. Yeldan I, Cetin E, Ozdincler AR. The effectiveness of low laser therapy on shoulder function in sub-acromial impingement syndrome. Disabil Rehabil. 2009;31:935-40.

17. Kelle B, Kozanoglu E. Low-level laser therapy and local corticosteroid injection in the treatment of subacromial impingement syndrome: a controlled clinical trial. Clin Rehabil. 2014. doi: 10.1177/0269215514520772.

18. Eslamian F, Shakouri SK, Ghojazadeh M, Nobari OE, Eftekharsadat B. Effects of low-level laser therapy in combination with physiotherapy in the management of rotator cuff tendinitis. Lasers Med Sci. 2012;27:951-8.
19. Pereira AN, Eduardo Cde P, Matson E, Marques MM. Effect of low-power laser irradiation on cell growth and procollagen synthesis of cultured fibroblasts. Laser Surg Med. 2002;31(4):263-7.

20. Almeida-Lopes L, Rigau J, Zangaro RA, Guidugi-Neto J, Jaeger MM. Comparison of the low level laser therapy effect on cultured human gingival fibroblasts proliferation using different irradiance and same influence. Lasers Surg Med. 2001;29(2):179-84.

21. Honmura A, Ishii A, Yanase M, Obata J, Haruki E. Analgesic effect of Ga-Al-As diode laser irradiation on hyperalgesia in carrageenin-induced inflammation. Lasers Surg Med. 1993;13(4):463-9.

22. Sakurai Y, Yamaguchi M, Abiko Y. Inhibitory effect of low-level laser irradiation on LPS-stimulated prostaglandin E2 production and cyclooxygenase-2 in human gingival fibroblasts. Eur J Oral Sci. 2000;108:29-34.

23. Rochkind S. Phototherapy in peripheral nerve regeneration: from basic science to clinical study. Neurosurg Focus. 2009;26(2):E8. doi:10.3171?FOC.2009.26.2E8.

24. Djavid G, Mehrdad R, Ghasemi M, Hasan-Zadeh H, Sotoodeh-Manseh G. In chronic low back pain, low level laser therapy combined with exercise is more beneficial than exercise alone in the long term: a randomised trial. Aust J Physiother. 2007;52:155-60.

25. Gur A, Karakoc M, Cevik R, Nas K, Sarac A, Karakoc M. Efficacy of low power laser therapy and exercise on pain and functions in chronic low back pain. Lasers Surg Med. 2003;32:233-8.

26. Hakguder A, Birtane M, Gurcan S, Kokino S, Turan F. Eficacy of low level laser therapy in myofascia pain syndrome: an algometric and thermographic evealuation. Lasers Surg Med. 2003;33:339-43.

27. Green S, Buchbinder R, Hetrick SE. Physiotherapy interventions for shoulder pain. Cochrane Database Syst Rev. 2003;Issue 2, Art. No: CD004258. doi: 10.1002/14651858.CD004258.

28. England S, Farrell A, Coppock J, Struthers G, Bacon P. Low laser therapy of shoulder tendonitis. Scan J Rheumatol. 1989;18:427-31.

29. Saunders L. The efficacy of low level laser therapy in supraspinatus tendinitis. Clin Rehabil. 1995;9:126-34.

30. Vecchio P, Cave C, King V, Adebajo AO, Smith M, Hazleman BL. A doubleblind study of the effectiveness of low level laser treatment of rotator cuff tendinitis. Br J Rheumatol. 1993;32:740-2.

31. Brukner P, Khan K. Clinical sports medicine. Sydney: McGraw-Hill; 2002.

32. Dogan SK, Saime AY, Ecvik D. The effectiveness of low laser therapy in subacromial impingement syndrome: a randomised placebo controlled double blind prospective study. Clinics. 2010;65:1019-22.

33. Abrisham MS, Kermani-Alghoraishi M, Ghahramani R, Jabbari J, Jomeh H Zare M. Additive effects of low-level laser therapy with exercise on subacromial syndrome: a randomised, double blind controlled trial. Clin Rheaumatol. 2011;30:1341-6.

34. Saunders L. Laser versus ultrasound in the treatment of supraspinatus tendinosis randomized controlled trial. Physiotherapy. 2003;89:365-73.

35. Taverna E, Parrini M, Cabitza P. Laser therapy versus placebo in the treatment of some bone and joint pathology. Minerva Ortopedica $E$ Traumatologica. 1990;41:631-6.

36. Stergioulas A. Low power laser treatment in patients with frozen shoulder: preliminary results. Photomed Laser Surg. 2008;26:99-105.

37. Bingol U, Altan L, Yurtkuran M. Low power laser treatment for shoulder pain. Photomed Laser Surg. 2005;23:459-64.

38. Bal A, Eksioglu E, Gurcay E, Gulec B, Karaahmet O, Cakci A. Low-level laser therapy in sub-acromial impingement syndrome. Photomed Laser Surg. 2009;27:31-6.

39. Thornton AL, McCarty CW, Burgess M. Effectiveness of low-level laser therapy combined with exercise program to reduce pain and increase function in adults with shoulder pain: a critically appraised topic. J Sports Rehabil. 2013;22:72-9.

40. Higgins PT, Altman DG. Assessing risk of bias in included studies. In: Higgins PT, Green S, editors. Cochrane handbook for systematic reviews of interventions, The cochrane collaboration. West Sussex England: Willey-Blackwell; 2008

41. Deeks JJ, Higgins PT, Altman DG. Analysing data and undertaking meta-analyses. In: Higgins PT, Green S, editors. Cochrane handbook for systematic reviews of interventions, The cochrane collaboration. West Sussex England: Willey-Blackwell; 2008.

42. GRADE Working Group. Grading quality of evidence and strength of recommendations. BMJ. 2004;328:1490-4. 